\title{
Correction of Postrhytidectomy Facial Asymmetry by Thread Lifting: A Case Series
}

\author{
AMR ABD EL-MONIEM EL-NAGGARY, M.D.* and AHMED F. El-SHERIF, M.D.** \\ The Department of Plastic Surgery, Faculty of Medicine, Fayoum University* and \\ Department of Plastic and Reconstructive Surgery, Faculty of Medicine, Ain Shams University**
}

\begin{abstract}
With the increased rates of rhytidectomy (face lift) surgeries performed, many patients present with the complaint of facial asymmetry due to relapse. The use of APTOS thread lifting for such patients could provide a simple, safe, and cost-effective alternative to redo surgery. In this work we describe our experience with the procedure. All patients exhibited significant satisfaction for the follow-up period of 3 months. Minimal adverse events were encountered and were majorly self-limited. Relapsed rhytidectomy patients with minor degrees of facial asymmetry are excellent candidates for facial thread suspension.
\end{abstract}

Key Words: Thread lift - Barbed - APTOS - Rhytidectomy Facial asymmetry.

\section{INTRODUCTION}

With an ageing population, the number of performed rhytidectomy (commonly referred to as 'facelift') procedures is substantially on the rise [1]. Rhytidectomy involves getting rid of facial wrinkles (rhytide $=$ wrinkle) to give a more youthful appearance. The changes that occur in facial skin with ageing vary according to the nature of the skin; thicker skin as that of the Asian population exhibits sagging and drooping, while thinner skin as that of Caucasians exhibits volume loss and wrinkling [2]. Consequently, a number of techniques are available within the arsenal of the plastic surgeon to mix and match from in order to achieve the most satisfactory outcome $[\mathbf{3 , 4 ]}$. The most commonly performed procedure, however, remains the superficial musculo-aponeurotic system (SMAS) flap or any modification of which ('traditional rhytidectomy', hereby on referred to as rhytidectomy).
This involves dissection beneath the superficial fascia of the face and neck, followed by separate tightening and re-draping of cervicofacial skin and the SMAS/platysma [1,2].

The use of barbed sutures for facial suspension has garnered attention in the recent couple of decades [5]. With the barbs on the thread acting as $\operatorname{cog}$ s that clutch and suspend a relaxed facial area, the technique offers a minimally invasive, timeand cost-effective alternative to rhytidectomy with faster recovery and less complications [6,7]. The term APTOS (anti-ptosis) was coined by Sulamanidze to describe barbed threads and was later popularized to include all products and technologies involved in facial thread lifting [8].

The ideal candidates for APTOS suspension are those with adequate soft-tissue volume who require a small lift or who are not fit for general anesthesia [5]. Another important group that may benefit from the procedure are those who have had some relapse after a previous rhytidectomy [6]. Redo surgery for such patients comes at a financial and rehabilitation cost, and thread lifting might offer a much needed alternative.

There is dire need for more studies on thread lifting. In a recent systematic examination of the literature [9], a gap of scientific data was highlighted regarding the procedure's outcomes and complications, and a need for reporting by diverse groups was indicated.

In this work, we aim to report our experience with APTOS thread lifting in terms of outcomes and safety in patients with postrhytidectomy facial asymmetry. 


\section{PATIENTS AND METHODS}

This retrospective analysis included patients that had previously undergone SMAS flap surgery and were presenting with some relapse on one side of the face. The patients who fit the inclusion criteria were given the choice of thread lifting or a redo surgery with a thorough explanation of the strengths and limitations of each procedure. Those who opted for the thread lifting procedure were asked to sign a written informed consent to be included in the study. The study adhered to the tenants of the Declaration of Helsinki.

The inclusion criteria were the presence of adequate soft tissue for thread concealment and the tolerability to local anesthesia. Patients were excluded if they gave history of multiple skin allergic reactions or infections, if they were immunocompromised, if they had metabolic imbalance, or if they had a propensity towards keloid / hypertrophic scar formation. The study spanned the period between June 2016 and June 2018.

All procedures were conducted by the same experienced surgeon (A.A.E.) in an outpatient clinic setting. We used the Visage Excellence Method packs purchased from APTOS (APTOS International, Tbilis, Georgia). Marking of the patient's face for needle insertion was conducted, followed by the application of local anesthesia $(0.25 \%$ solution of lidocaine with epinephrine 1 ; 100,000). Under complete aseptic conditions, the needle was inserted with appropriate consideration to vector and facial expression lines, it was then advanced in a sinuous manner with the tip being moved from side to side. Care was taken to maintain the depth of the needle at a constant level just above the fat layer. Upon reaching the target point, the needle was then turned and reinserted until it appeared on the opposite side. Following insertion of all the barbs in the skin, assessment of facial symmetry was conducted by 2 members of the surgical team. When deemed satisfactory, the ends of the suture threads were cut using surgical scissors. Any remaining protrusions were concealed by skin massage.

Following the procedure, the patients were asked to avoid strenuous activity for the following 2 days, with promotion of head lifting to minimize swelling. The patients were prescribed postoperative antibiotics (Co-amoxicalv 500mg twice daily for 5 days) to minimize the risk of infection and an oral analgesic (Ibuprofen 500mg twice daily for 5 days). The follow-up schedule was 7 days, 1 month, and 3 months postoperatively.

On each follow-up visit, the patient was photographed. A modified visual analogue score (VAS) was used to rate the patient's satisfaction with the facial symmetry. The VAS score was also obtained preoperatively before the procedure. Any complaints or complications were documented.

Data were tabulated and statistical analysis was conducted using SPSS v20 (IBM, USA).

\section{RESULTS}

Sixteen patients were included in this study. Those were fourteen female patients $(87.5 \%)$ and two male ones (12.5\%). The mean age of the cases was 46.3 years (range, 37 to 52 years). The mean preoperative VAS score for satisfaction with facial symmetry was $2.5( \pm 0.8 \mathrm{SD})$. The mean time from the previous rhytidectomy surgery was 5.5 months (range, 2 to 9 months).

Figs. (1-4) depict the immediate postoperative aesthetic improvement in symmetry seen in a sample of our patients after one week postoperatively. The mean VAS score at such period was $8.5( \pm 2.6)$. This change was highly statistically significant when compared to preoperative scores $(p<0.0001)$. The satisfaction level was maintained after 1 month postoperatively with the mean score increasing to $8.8( \pm 3.1)$ and continued as such at 3 months post-

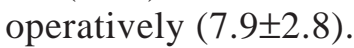

Regarding the safety of the procedure, the most commonly encountered complication was erythema that occurred in 7 patients $(43.8 \%)$. This resolved spontaneously in all patients.

Three patients (18.8\%) experienced skin dimpling, and in one of them $(6.3 \%)$ there was associated facial stiffness. Both conditions resolved spontaneously. No patients experienced infection. Only one patient (6.3\%) had thread resurfacing at 1 month postoperatively that required removal in the same direction of insertion. 

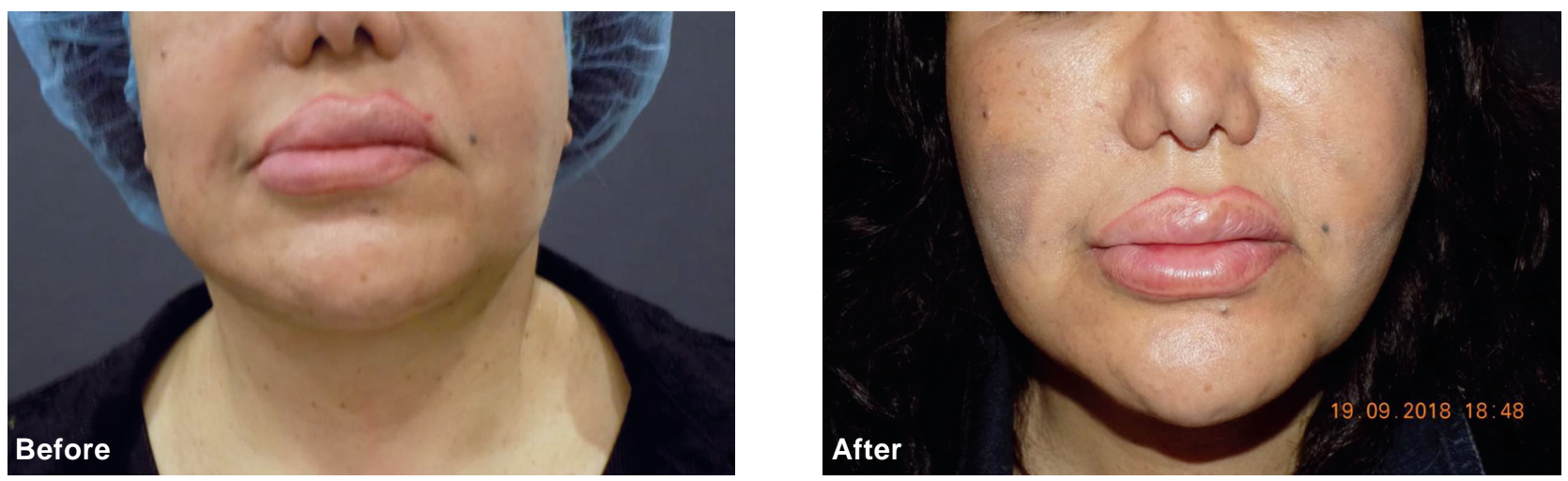

Fig. (1): Before and after frontal lower face images of one of our patients taken preoperatively and at 1 month postoperatively.
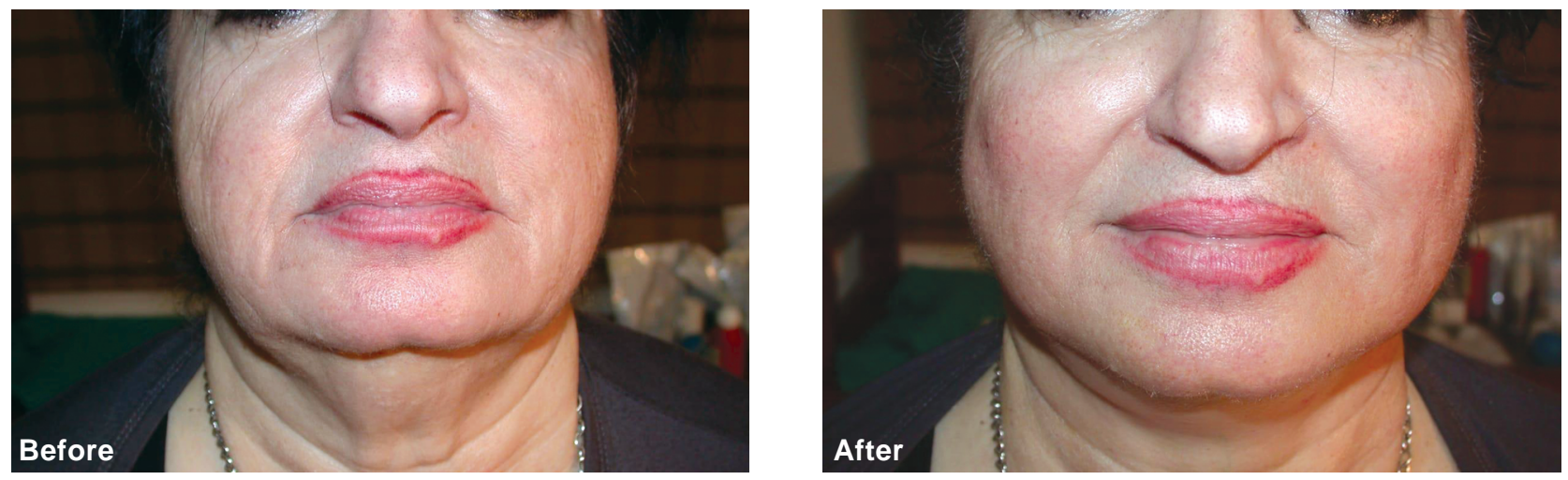

Fig. (2): Before and after frontal view images of midface of one of our patients taken preoperatively and at 3 months postoperatively.
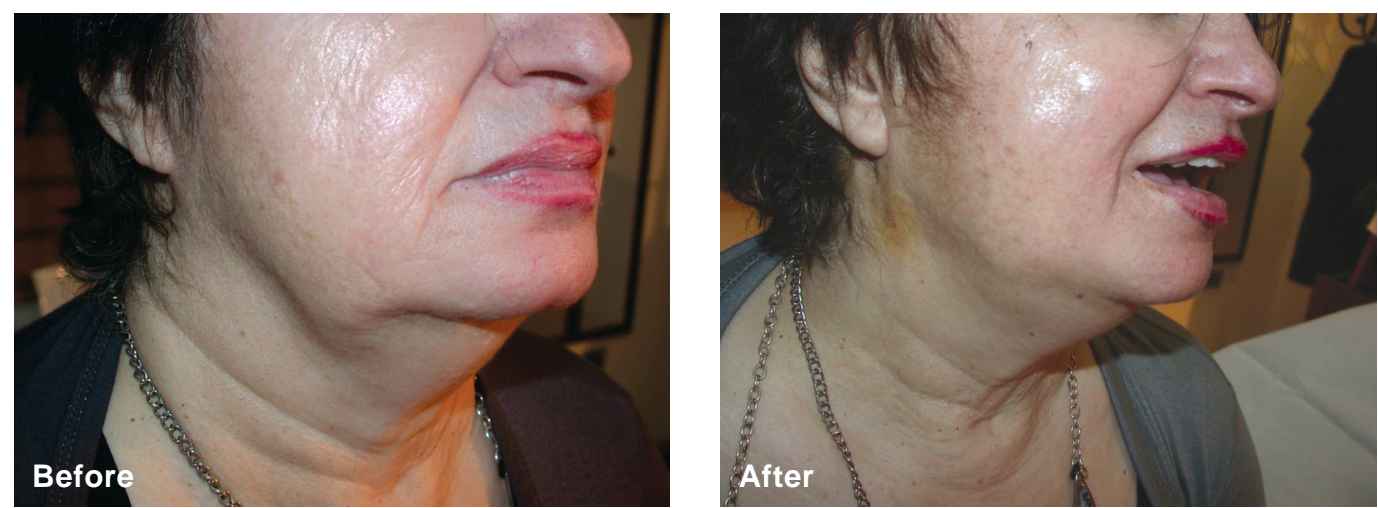

Fig. (3): Before and after lateral view (right side) images of lower face of one of our patients taken preoperatively and at 1 month postoperatively.
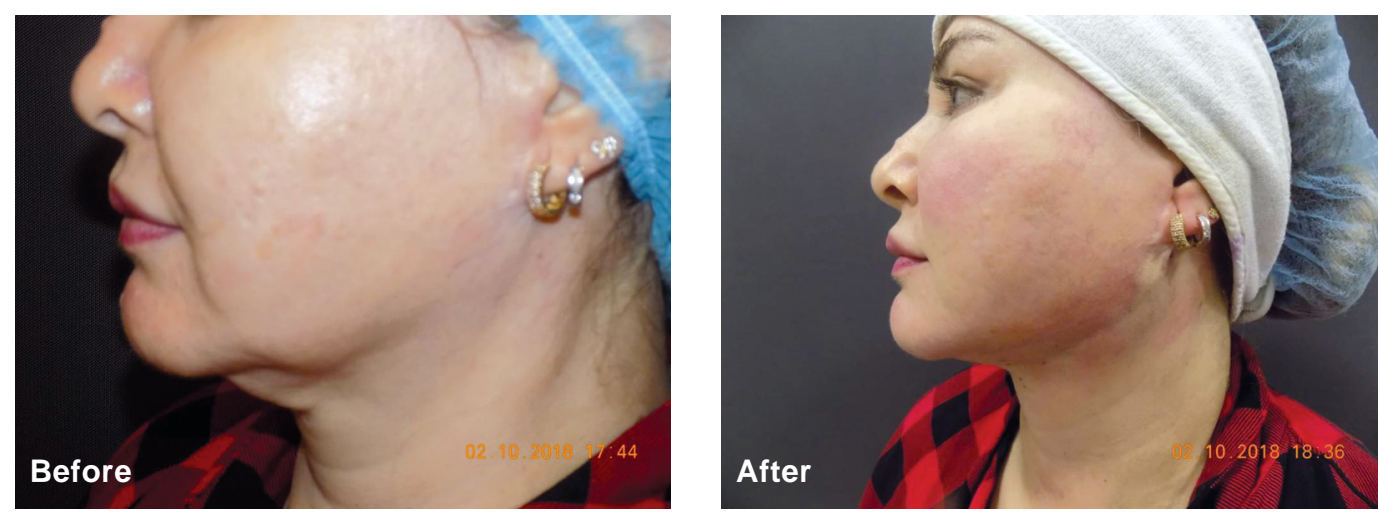

Fig. (4): Before and after lateral view (left side) images of lower face of one of our patients taken preoperatively and at 1 month postoperatively. 


\section{DISCUSSION}

In this work, we report on the use of APTOS thread lifting as a simple outpatient procedure for patients complaining about postrhytidectomy facial asymmetry. Our results reveal that the procedure is safe and effective with satisfactory outcomes as perceived by the patients.

To the best of our knowledge, this is the first report in the literature dedicated to facial thread lifting following rhytidectomy relapses on one side of the face.

In the recent review by Sarcu and Adamson [10], the psychology of the facelift patients was examined. More emotional concern about minor changes in facial features exists in this group of patients than in the general population. Half of the patients exhibit anxiety postoperatively. This might explain the low threshold of tolerance towards any asymmetry observed following rhytidectomy and the immediate seeking of surgical consultation. In our patients, the low mean VAS score $(2.5 / 10)$ for satisfaction with facial symmetry reflects such findings.

Outcomes of facial thread lifting vary in the literature. In the review conducted by Villa et al., back in 2006 [7], only six studies were found for inclusion in the review. Data from those studies revealed a good safety profile with minor adverse effects, but results on efficacy of the procedure, peak correction, and longevity were lacking. A second systematic review was conducted by Gülbitti and colleagues in 2017 [9]. Only 6 more studies on the subject were added to the literature over the span of the decade. The authors again concluded that outcome data were lacking and that patients needed to be educated about the possibility of the procedure being short-lived. Our data suggests high degrees of satisfaction among patients extending up to 3 months postoperatively. Our followup data were not gathered beyond that so we cannot fully comment on longevity, but all patients were counselled on the possibility of short-lived results and still opted for the procedure. This could be attributed to the relative inexpensiveness of the procedure when compared to traditional face lifting [5].

In the comparative analysis by Abraham and colleagues [11], the effect of thread lifting alone was considered inferior to a rhytidectomy or a combined procedure and was attributed mainly to postoperative edema and inflammation. Our data suggests otherwise, with the satisfaction levels maintained up to one month postoperatively when all the edema and inflammation had resolved. The thread material used in the mentioned study was different than the one used in our study. Furthermore, patient selection might play an important role. Our work was concerned with thread use in patients that had already undergone surgical facial rejuvenation and who had minor degrees of asymmetry, amendable to thread suspension. This is different when compared to other studies that deal with primary face lifts using threads. In the latter studies, soft tissue remodeling is not a component and might explain the mediocre results.

The adverse effects that we encountered were similar to those reported elsewhere in the literature $[6,9,12]$. Most complications were self-limited and did not require any intervention. Erythema was the most commonly encountered complication $(43.8 \%)$ but was not present in any patient at or beyond one month postoperatively. We had a $0 \%$ infection rate; this could be attributed to our routine use of postoperative systemic antibiotics to minimize any infection risk, contrary to no use mentioned in multiple other reports.

In conclusion, the use of APTOS threads for correction of postrhytidectomy facial asymmetry is safe and effective, and provides patients with a minimally invasive, quick, and relatively cheap alternative to redo surgery. Patient counselling about available options and the limitations of each option is imperative. Further studies with longer follow-up are needed to examine sustainability of the procedure.

\section{REFERENCES}

1- Pourdanesh F., Esmaeelinejad M., Jafari S.M. and Nematollahi Z.: Facelift: Current concepts, techniques, and principles. In: A textbook of advanced oral and maxillofacial surgery Volume 3. In Tech., p. 653-79, 2016.

2- Carniol P.J. and Gane D.T.: Is there an ideal facelift procedure? Curr. Opin. Otolaryngol. Head Neck Surg. Sug., 15 (4): 244-52, 2007.

3- Wang R., Yang J., Guo K., Zhong A., Tong J., Xiong L., et al.: Asian facelift technique refinement with high patient satisfaction: FACE-Q report. Ann. Plast. Surg., May Preprint, 2018.

4- Tyu M-H., Khoo L.S., Lee J.O. and Li D.: Reframing the etiology of facial sagging from a facelift perspective. Plast. Reconstr. Surgery Glob Open. Jul., 6 (7): e1831, 2018.

5- Betossi D., Botti G., Gualdi A., Fundaro P., Nocini R., Pirayesh A., et al.: Effectiveness, longevity, and complications of facelift by barbed suture insertion. Aesthetic Surg. J. Feb., Preprint, 2018.

6- Kalra R.: Use of barbed threads in facial rejuvenation. Indian J. Plast. Surg. Off Publ Assoc. Plast. Surg. India. Oct., 41 (Suppl): S93-100, 2008. 
7- Villa M.T., White L.E., Alam M., Yoo S.S. and Walton R.L.: Barbed sutures: A review of the literature. Plast. Reconstr. Surg. (Internet). Mar., 121 (3): 102e-108e, 2008.

8- Sulamanidze M. and Sulamanidze G.: APTOS suture lifting methods: 10 years of experience. Clin. Plast. Surg. Apr., 36 (2): 281-306, 2009.

9- Gülbitti H.A., Colebunders B., Pirayesh A., Bertossi D. and van der Lei B.: Thread-lift sutures: Still in the lift? A systematic review of the literature. Plast. Reconstr. Surg., Mar., 141 (3): 341e-347e, 2018.
10- Sarcu D. and Adamson P.: Psychology of the facelift patient. Facial Plast. Surg., Jun., 33 (3): 252-9, 2017.

11- Abraham R.F., DeFatta R.J. and Williams E.F. $3^{\text {rd.: }}$ Thread-lift for facial rejuvenation: Assessment of longterm results. Arch. Facial Plast. Surg., 11 (3): 178-83, 2009.

12- Sulamanidze M., Sulamanidze G., Vozdvizhensky I. and Sulamanidze C.: Avoiding complications with aptos sutures. Aesthetic Surg. J. Nov., 1; 31 (8): 863-73, 2011. 